\title{
Dynamical and Topological Aspects of Consensus Formation in Complex Networks
}

\author{
A. Chacoma, M. N. Kuperman, and G. Mato \\ Centro Atómico Bariloche (CNEA) and Instituto Balseiro and \\ Consejo Nacional de Investigaciones Científicas y Técnicas, \\ (8400) Bariloche, Río Negro, Argentina.
}

\begin{abstract}
The present work analyses the propagation and degradation of information transmitted across different complex networks topologies. The degradation of the information, coded in simple messages, is the consequence of the inclusion of errors during the transmission and manifest itself in two different ways. The first one results in some addresses getting a wrong message, but maintaining the global amount of information in the system. The second one consists in the collapse of the global information into a single message. We analyze how the topology of the underlying networks promotes or inhibits the degradation process.
\end{abstract}

\section{INTRODUCTION}

The role of complex networks as a mathematical tool to formalize the underlying topology in several propagating phenomena of social extraction has been well established in the last years. When looking for models of the spread of diseases, the propagation of information, rumors or ideas, complex network provide a plethora of alternative topologies that serve to mimic the complex weave of the interpersonal relationships [1, 3-5]. At a more abstract level, diffusion in general is one of the fundamental processes taking place in networks [6-8]. Diffusive propagation on a network generates the opportunity that agents get in touch and interchange ideas and information. Given the appropriate rules for interchange and network topology this could lead to the appearance of consensus among the opinions of the different agents.

Consensus formation is a widely studied in social sciences. One of the main goals is to understand the emergence of a system consensus involving a number of interacting agents [26]. Thus problem has been studied analytically, for instance, in [27-29], where it is proposed that each agent alters its opinion according to some weighted average of the rest of system. It is found that if all the recurrent states of the Markov chain communicate with each other and are aperiodic, then a consensus is always reached. In most of the existing models the update of opinions takes place via a linear mechanism [30, 31, 33]. Moreover, they do not usually analyze the influence of the network topology. Although see, for instance, [26], where a complex network appears as a susbtrate of a set of agents with linear dynamics and [32], where the topology of the network is altered by the interactions between the agents.

The structure of the complex networks has been extensively studied from the point of view of transfer of information. A lot of emphasis has been put on the influence of topology, studying for instance whether scale free networks are more efficient than regular ones (ref). In this context it has been shown, for instance, that it is very important whether the network consists of homoge- neous nodes or it has a structure of routers and peripheral nodes (ref). Another important aspect is the clustering, because the presence or absence of loops can affect information transfer (ref). For instance, in networks with modular structures, it has been shown that the velocity of the information propagation depends non linearly on the the number of modules. A piece of information will propagate faster for networks having either a small number or a large number of modules [9].

A set of interacting agents on a network can give rise to a dynamically changing local environments where the process of interchange of opinions take place. In this paper we focus on three aspects: 1) How the local interaction rules control the convergence to consensus, in particular we analyze both linear and non-linear rules. 2) What is the influence of dynamics of the agents in the network. We first consider the the propagation of the information by considering a naïve strategy for neighboring node selection. If the target node is not among the neighbors, a neighbouring node is selected at random for the trajectory of the agent. Then we consider a preferential choice strategy, where the agents are more likely to move to more connected nodes. 3) What is the effect of the different parameters of of the network topology, such as clustering or assortativity. In the following sections we present the model in more details and a description of our results.

\section{NETWORK TOPOLOGIES}

Throughout this work we have used several families of networks with different topologies and algorithmic constructions, though always containing the same number of nodes and links.

Regular Small World networks: We consider first regular networks with a tuned degree of disorder, and consequently different degrees of clustering and mean distance. This networks were built using a modified algorithm based on the originally proposed in [17] to constrain the resulting networks to a subfamily with a delta shaped degree distribution. We call this family of net- 
works the k-Small World Networks ( $k$-SWN) [18], where $2 k$ indicates the degree of the nodes. The building procedure starts with an ordered regular network whose order is broken by exchanging the links of two pairs of connected nodes in a sequential way. Starting for example from an ordered ring network, each link is subject to the possibility of being exchanged with probability $p_{d}$. If the exchange is accepted, we randomly choose another link to switch the partners in order to get two new pairs of coupled nodes. Double links are always avoided, thus if there is no way to avoid a double link with the present selection of node, a new choice is done. In this way all the nodes preserve their degree while the process of reconnection assures the introduction of a certain degree of disorder. It must be stressed that the dependence with $p_{l}$ of the clustering coefficient and path length is qualitatively similar to the one is observed as a function of $p_{l}$ in Small World Networks [17]. In this way we can evaluate the effects of clustering and distance independently of the degree distribution.

Small World Networks: To include the possible effects of the degree distribution on the analyzed dynamics we consider then, the usual algorithm described in [17], where only one link is rewired at a time, maintaining the attachment to one of the adjacent nodes and randomly connecting the other extreme. These networks not only posses different degrees of clustering and mean distance but also different binomial degree distributions, linked to the disorder parameter $p_{l}$. We we refer to this networks as SWN.

Scale free networks: Finally, we are interested in studying networks with a degree distribution closely related to those frequently observed in real contexts. Thus we consider Scale Free Networks (SFN) [19] with different degrees of assortativity, following the prescription presented in [20] with a slight modification to obtain networks with tunable positive or negative assortativity. In order to get positive assortativity, the algorithm consists in departing from a random network with the desired degree distribution and sequentially rearranging the nodes at the ends of a pair of randomly chosen links. At each step two links of the network are chosen at random. The corresponding four nodes are ordered with respect to their degrees and the original links are deleted. Then, with probability $p_{a}$, a new link connects the two nodes with the smaller degrees and another connects the two nodes with the larger degrees. Otherwise, the links are randomly rewired. As double connections are forbidden, if the rewiring indicates connecting already connected nodes, the change is discarded and the original links are reestablished. If instead of connecting the nodes with the highest and lowest degrees between them we connect the node with the highest degree with the node with the lowest degree, and the other two between them we obtain networks with negative assortativity. The parameter $p_{a}$ governs the final degree of assortativity.

\section{Incluir una figura}

\section{DYNAMICS OF CONSENSUS FORMATION}

We have $N_{p}$ agents that travel from an initial node to a final node of the network. These nodes are chosen randomly with uniform distribution among the $N$ network nodes. Each agent has an opinion that is indexed by an integer number between 1 and $N_{c}$. In the initial condition the opinion of each agent is chosen randomly with the constraint that there is each opinion appears the same number of times. The number of agents is given by $N_{p}=10 N$. The number of nodes is in the range $[1000,10000]$.

At a given time there is some number of agents in a given node. If there are $N_{i}$ agents in node $i$, each of of them is assigned and index $j=1, \ldots, N_{i}$ according to the order of arrival, with earlier arrivals having lower indices. The node with the lowest index checks whether the node where is located is a neighbor of its destination node. If this is the case it moves in that direction and its movement stops. If not, it chooses one of its neighbors following one of two possible strategies. In one case, the node is randomly chosen among those conforming the neighborhood. The other strategy requires that each node has information about the degree of all its neighbors. The one with the higher degree is chosen, according to the preferential choice strategy [12] that a priori optimizes the possibility of reaching the target following a shorter path. This procedure is repeated in random order for all the nodes of the network.

This second strategy is motivated by the need to optimize the ability to efficiently navigate and search in a network without a thorough knowledge of its topological properties. As we are interested in finding the path to a target node from an initial one using only local information, we appeal to the concept of random walk centrality [10] $(C)$, that characterizes those nodes which will be much frequently visited by initially uniformly distributed random walkers. Another interesting property is that when we consider the set of all possible random walks between two nodes, the one reaching the node corresponding to a larger value of $C$ is faster than the others. After finding that for a Barabási-Albert scale-free network [19], the degree of a node is directly related to its centrality the maximum degree strategy was proposed in [11]. In this case, each node must have information on its neighbors degree so that the a walking agent always moves to the neighboring node having the highest degree. A relaxed version of this strategy was also proposed in [12], where the authors also study the preferential choice strategy, in which the node with the larger degree has the higher probability to be chosen. This strategy is equivalent to the former one when the probability is 1 . As we will further show, these two strategies, despite that being 
optimal for network navigation, result in different results for consensus formation.

\section{Local interaction between agents}

We propose the following rules for the interaction between agents. The change of opinion will happen with probability $\rho$. In the present study we analyze two possibilities, namely:

- Linear: An agent, instead of keeping its original opinion, takes a different one, randomly chosen among the opinions of the other agents in the same node.

- Quadratic: An agent, instead of keeping its original opinion, takes two opinions randomly chosen among the one of the other agents in its node, If they are the same, then this will be the new opinion, otherwise, the original opinion is kept.

\section{Characterization of consensus formation}

These mechanisms for interaction lead to a global modification of the probability distribution of the opinions. This effect can be quantified using the Kullbak-Leibler distance [23]. Given two probability distributions $P$ and $Q$ it is defined by

$$
D_{K L}=\sum_{k} P(h) \log \frac{P(h)}{Q(h)}
$$

If the initial distribution is $Q(h)=1 / N_{c}$ then the maximum value the Kullback-Leiber distance can take is when the distribution $P(h)$ is concentrated in only one point: $P(h)=\delta_{h, h_{0}}$, in which case $D_{K L}=\log \left(N_{c}\right)$. In other words consensus is maximized when $D_{K L}$ increases. Let us remark that the probability distribution is measured over the agents that have reached their target destinations.

\section{Probability distribution of contents in the linear case}

We now prove that in the linear case the interactions between agents cannot alter the distribution probability of opinions. Let us denote by $P(k)$ the probability of having an agent with opinion $k$. The probability that this opinion is altered from $k$ to $l$ is $P(k \rightarrow l)=\rho P(l)$. Similarly $P(l \rightarrow k)=\rho P(k)$. The probability of having an agent with opinion $k$ (resp. $l$ ) in the first position of the node is $P(k)$ (resp. $P(l)$ ). Therefore the number of agents that change their content from $k$ to $l$ is in average $N_{p} P(k) P(k \rightarrow l)=N_{p} \rho P(k) P(l)$ that is exactly the number of opinions that change from $l$ to $k$. This implies that the Kullback-Leibler distance should not be affected by the interaction and consensus can never be achieved.

\section{NUMERICAL RESULTS}

Although different network topologies were used throughout our simulations, all of them consisted in $N=10000$ nodes and a mean degree $K_{m}=6$. We will discuss each topology separately and contrast the obtained results whenever the comparison is relevant.

\section{Terminated dynamics}

$$
k-S W N
$$

As mentioned before, we took $N=10^{5}$ an $K_{m}=K=$ $2 k=6$, and with the disorder parameter $p_{d}$ ranging between 0 and 1 . In order to characterize the formation of consensus we started by analyzing the change in the initially uniform distribution of messages. As expected, when the underlying dynamics is linear, there is a slight departure from the initial distribution due to unavoidable fluctuations. On the contrary, when nonlinear dynamics are considered the situation changes dramatically. This can be observed in Fig.(1), where we show the final distribution of opinion for the linear and quadratic dynamics, for two limiting extreme cases, $p_{d}=10^{-3}$ and $p_{d}=.9$
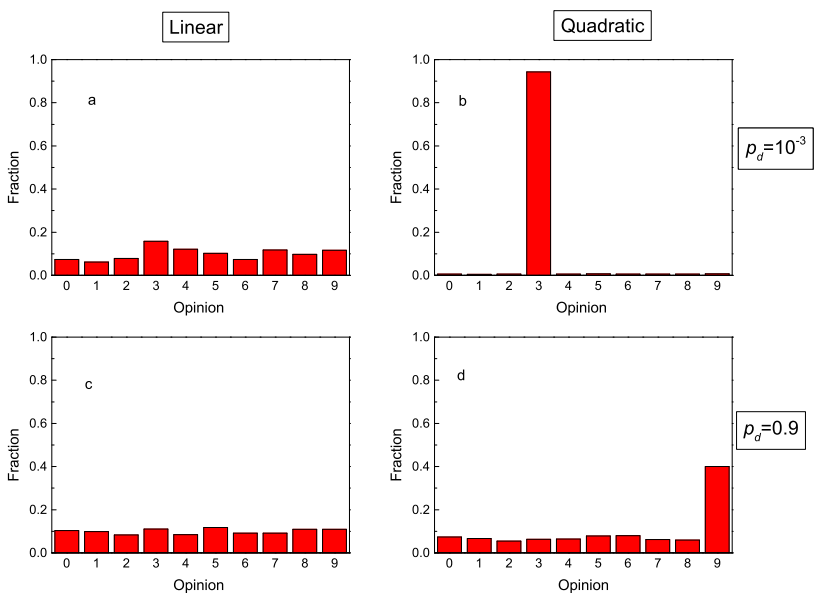

FIG. 1: Final distribution of opinions for two different dynamics and two disorder parameters of $k$-SWN's: a) Linear, $p_{d}=10^{-3}$, b) Quadratic, $p_{d}=10^{-3}$, c) Linear, $p_{d}=0.9, \mathrm{~d}$ ) Quadratic, $p_{d}=0.9$

As mentioned before, the departure of the distribution of opinions from the initial one can be measured through the $\mathrm{K}-\mathrm{L}$ distance, As the network disorder increases, the $\mathrm{K}-\mathrm{L}$ distance decreases. One obvious reason is that as the mean path between nodes is smaller for disordered 
networks, a given agent takes less time to reach its target. This effect can be observed in Fig. 2a, where the values of the $\mathrm{K}-\mathrm{L}$ distances is plotted against the average path length $L$. However, $L$ is not the only quantity that changes as a function of $p_{d}$. The clustering coefficient $C$ also decreases as the disorder grows and can be affecting the behavior of the K-L distance. The plot in Fig $2 \mathrm{~b}$ shows the change in the $\mathrm{K}-\mathrm{L}$ distance as a function of $C$. In both cases we observe two different regimes with slow and fast rates of change respectively. The fast change regime in Fig. 2a corresponds to a fast change in $C$ as a function of $L$. The analogous situation is observed in Fig. 2b. We conclude that not only the change in path length contribute to the difference in the K-L distance but also the change in clustering.

\section{$S W N$}

While the construction of SW networks is similar to its regular version and also presents an analogous behavior in terms of the values of $C$ and $L$ as a function of the disorder coefficient, there exists an apparent difference. The degree distribution of these networks changes from deltiform to binomial at the moment of introducing the slightest disorder. The associated binomial distribution is given by [24]

$P(h)=\sum_{n=0}^{\min [h, k]} C_{k}^{n}\left(1-p_{d}\right)^{n} p_{d}^{k}-n \frac{\left(k p_{d}\right)^{h-k-n}}{(h-k-n)} \exp \left(-k p_{d}\right)$

where $k=K_{m} / 2$ and $K_{m}$ is the mean degree of the network.

So, in addition of the effects of the topology on the formation of consensus due to the change of $C$ and $L$ we need to consider the effects attributed to the change in the degree distribution. As in the previous case, we observe a transition towards consensus that can be much beter quantified by studying the behavior of the K-L distance. The plots in Figs. 3a and $3 \mathrm{~b}$ show the dependence of the K-L distance on $C$ and $L$. In this case, we observe that the growth of the K-L distance behaves differently than the analogous situation in $k$-SWN for the quadratic dynamics, the one that promotes consensus formation. This difference could be due to the combined effect of an increase in $L$ and a change in the degree distribution.

Indeed, if we compare both families of networks in a unique plot it is more evident that the spreading of the degree distribution goes against the formation of consensus. We show this in Fig 4, where we contrast the values of the K-L distance for the same values of clustering $C$ and mean path length $L$ for both types of networks. The figure shows that for the same values of clustering or path length, SWN networks start to fail in reaching consensus once the degree distribution has adopted a clear binomial profile, corresponding to lowest values of $C$ and $L$.

\section{SF Networks}

So far we have studied SW networks with different degrees of disorder. The difference between SWN and $k$-SWN evidenced the effect of the heterogeneity of the degree distribution on the dynamics, that goes against the formation of consensus. SF networks, that can be considered as disordered networks present a particular degree distribution. We choose to analyze the behaviour of the consensus emergence on these networks and compare it with our previous results. But SF networks are not only characterized by its degree distribution. Among other properties, two networks might have the same degree distribution but present different assortativities.

The assortativity a can be measured as the Pearson correlation coefficient of degree between two neighbouring nodes [25].

$$
a=\frac{\sum_{j k}\left(A_{i j}-k_{i} k_{j} / 2 m\right) k_{i} k_{j}}{\sum_{j k}\left(k_{i} \delta_{i j}-k_{i} k_{j} / 2 m\right) k_{i} k_{j}}
$$

where the sumation is over each pair of nodes $(i, j)$. A is the adjacency matrix and $k_{n}$ se is the degree of node $n$. When this coefficient is positive indicates a higher occurrence of links between nodes of similar degree, while a negative value reveals relationships between nodes of different degree.

We choose to study the effect of this quantity in order to unveil the role of hubs in the obtained results. The calculated values of the K-L distance are low, as expected from previous results with highly disordered SW networks. An interesting phenomenon is observed as a function of the assortativity. We find that the optimal result with respect ot formation of consensus corresponds to highly assortative or disassortative networks. This can be observed in Fig.5

\section{Transient dynamics}

Most of the features observed once the totality of agents have arrived to the target can be explained by analyzing the transient dynamics

\section{Distribution of walking length}

The process of consensus formation requires that the agents change their opinion in their way to their target destination. By studying the amount of steps a given agent needs to make from the origin to the destiny and 


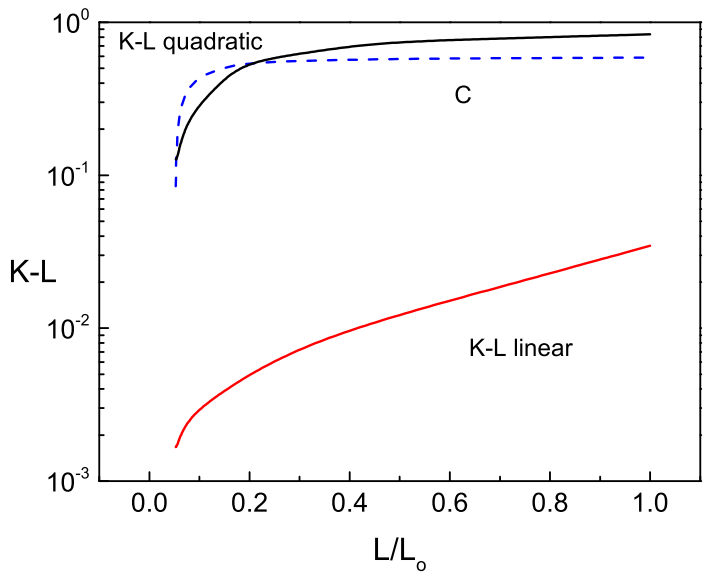

(a)

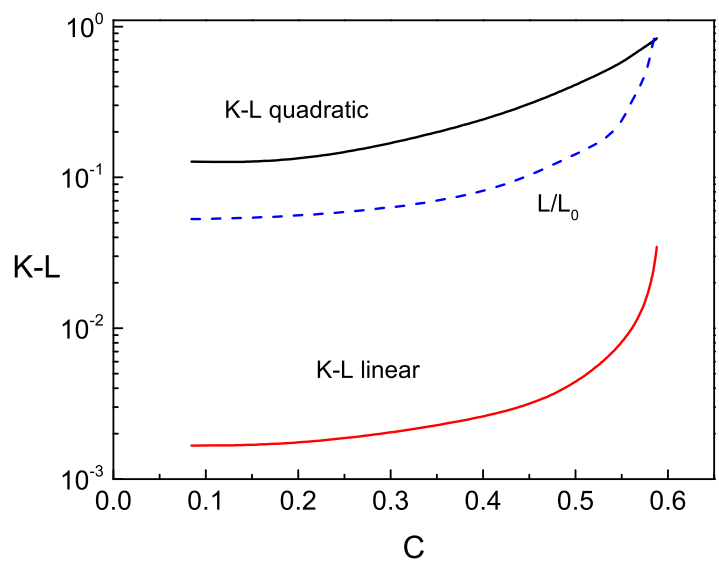

(b)

FIG. 2: K-L distance for different values of $p_{d}$ on $k$-SWNs and with linear and quadratic dynamics. a) as a function of $L / L_{o}$, with $L_{o}$ the mean path length of the ordered network. The dashed line is the value of $C$; b) as a function of $C$. The dashed line is the value of $L / L_{o}$.

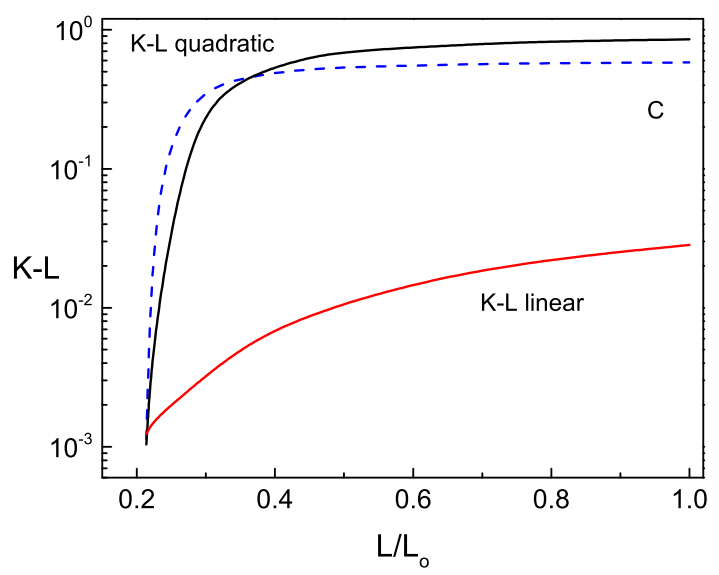

(a)

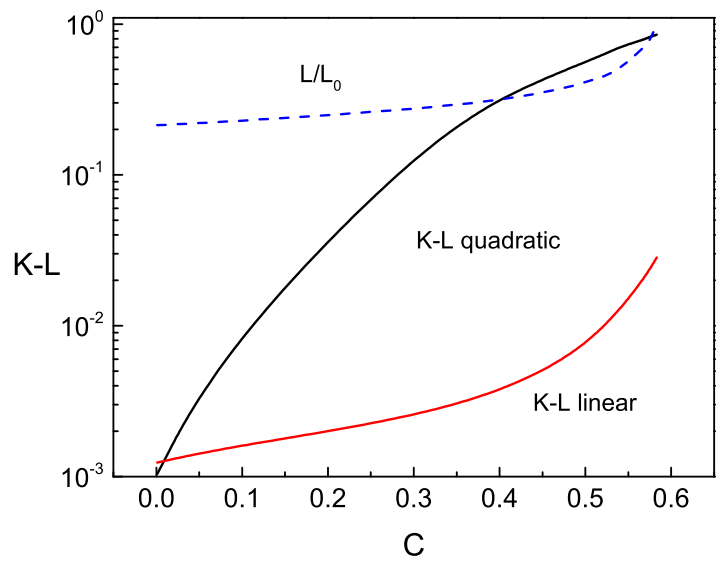

(b)

FIG. 3: K-L distance for different values of $p_{d}$ on SWNs and with linear and quadratic dynamics. a) as a function of $L / L_{o}$, with $L_{o}$ the mean path length of the ordered network. The dashed line is the value of $C ; \mathrm{b}$ ) as a function of $C$. The dashed line is the value of $L / L_{o}$.

knowing the probability of change of opinion at each step we can estimate the probability that an opinion has been altered. Fig. 6a shows the distribution of the number of steps (or walking length) for $k$-SWN and SWN of different disorder degree. In Fig. $6 \mathrm{~b}$ we plot the same for SF networks. As can be observed, there is a dramatic reduction in the number of required steps as the network gets more disordered. The curves corresponding to highly disordered $k$-SWN and SWN are very similar to those observed for SF networks. Besides, the plot corresponding to Sf networks shows a non monotonic dependence of the assortativity. These result is consistent with what have been already shown in the previous section. Consensus formation is harder when the underlying network is only barely dissortative.

These results are independent on the choice of the dynamics. Same aspect of our results can be understood by observing the distribution of walking length. The higher the number of steps, the higher is the probability of opinion changes and to get a total consensus.

The distribution of walking length is directly linked to the performance of the networks when defining the 


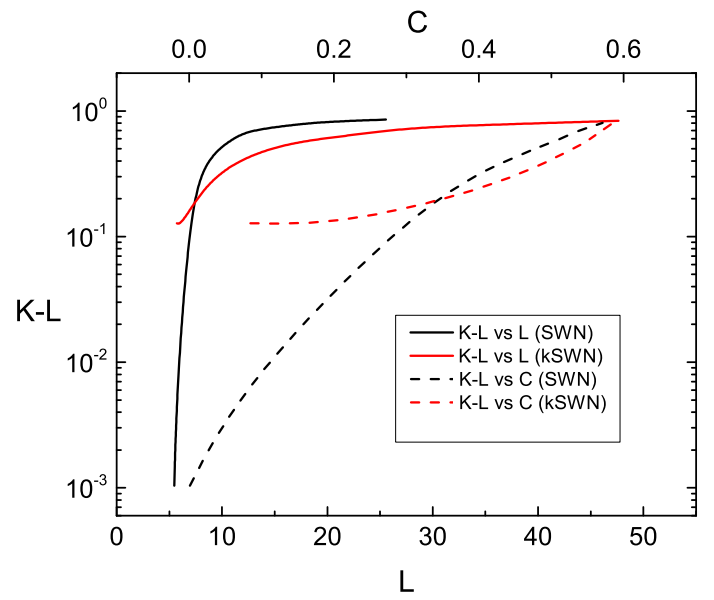

FIG. 4: Comparison of the K-L distance as a function of $L$ (lower $x$-axis,) and $C$ (upper $x$-axis).

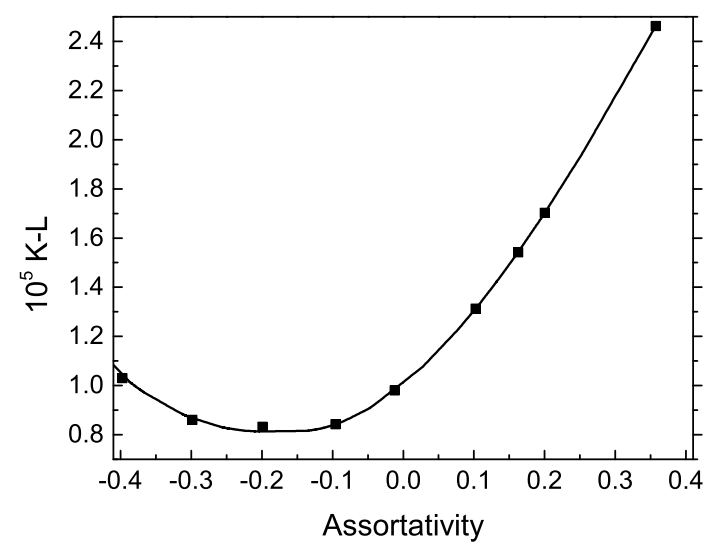

FIG. 5: K-L distance for different values of assortativity $a$ on $\mathrm{SF}$ networks with quadratic dynamics.

underlying paths topology. But as we will show in the following discussion, it is not the only relevant feature.

\section{Transient length}

The transient dynamics still has more information to reveal. So far we have only taken into account the number of steps. Some of these steps are made simultaneously by different agents. The total time taken by the whole process to end must be equal to the time taken by the agent linked to the maximum number of steps. In Figs $7 \mathrm{a}$ and $7 \mathrm{~b}$ we show the cumulative amount of agents that reached destination as a function of time.

These plots reveal an interesting feature, that we have highlighted in Fig 8. We observe that all the curves col- lapsed when the time is scaled with a factor $t_{s}\left(p_{d}\right)$ or $t_{s}(a)$ that depends on the topology of the underlying network. The only curve that departs from the rest is the corresponding to the assortative SF network (dashed in the plot), a fact that could be clearly observed in the previous plot.

\section{Buffer dynamics}

From the moment the agents start to reach their destination, the initial distribution of the number of agents in the nodes changes its shape, starting from a Gaussianlike distribution to turn into one with a high number of nodes with a low number of agents and a few very with high number. The rate of change of this distribution depends on the topology of the network, but if we analyze this distribution as a function of the amount of arrived agents instead of the spent time we find that for $k$-SWN they are almost the same, while this not the case for SWN. Fig 9a shows the distribution of tje number of agents for several networks. The curve corresponding to all the $k$-SWn and to the SWN with $p_{d}=10-3$ are superposed. On the contrary, SWN with different disorder degrees present different behaviors. The last is also true for for SF networks. as shown in Fig 9b

\section{Opinion change dynamics}

A natural question is at what stage of the dynamics occur the most part of the change of opinions. For that, we have recorded the rate of change occurrence and plotted it against the amount of agents that reached destination. While $k$-SWN and SWN show the same dynamics for low values of $p_{d}$, they are very different for higher disorder degrees. This is shown in Fig 10a In highly disordered $K$-SWN the changes occur at the beginning of the dynamics (high number of mobile agents), while for SWN it is just the opposite. I turn, SF networks behave as highly disordered SWN, as shown in Fig 10b.

\section{DICUSSSION}

-Summary of results

-We have also considered some alternatives rules:

- Higher order $n$ : As in the previous case, taking $n$ opinions from the other opinions in the node.

- Poissonian: The new opinion is take form another agent whose index is chosen randomly number of messages from a Poisson distribution. The mean value of the distribution is $\mu=2$. 


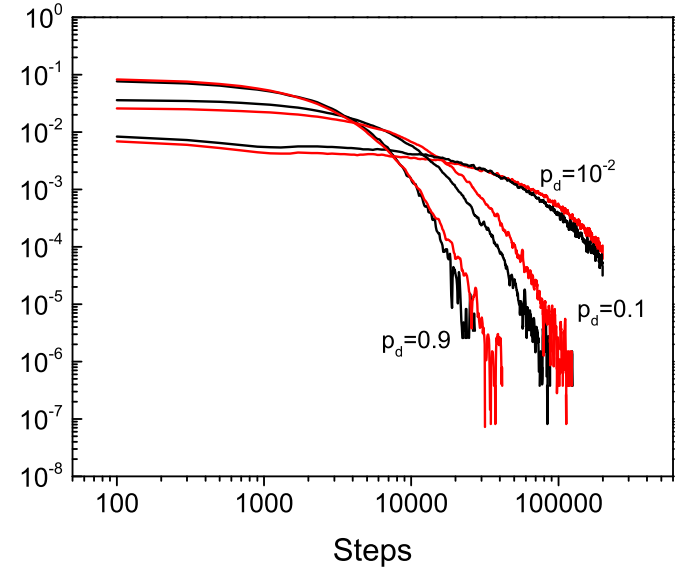

(a)

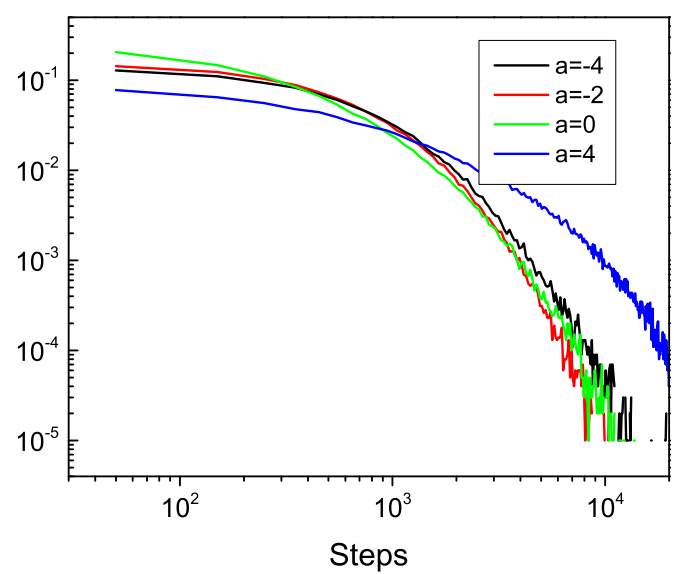

(b)

FIG. 6: Step distribution for a) k-SWN (black) and SWN (red), and b) SF networks

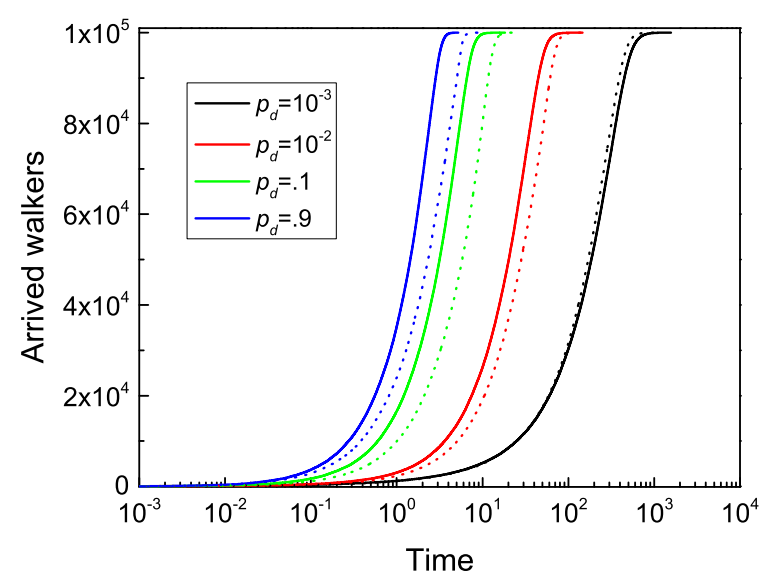

(a)

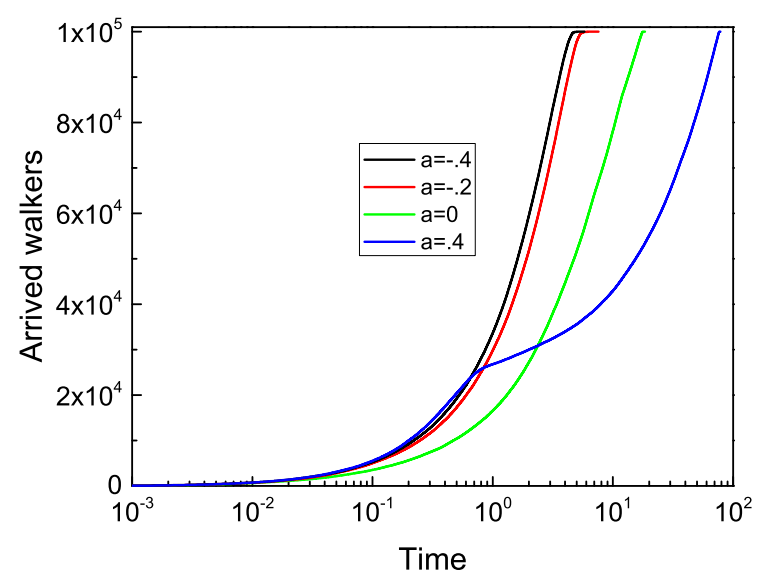

(b)

FIG. 7: Cummulative fraction of arrived agents as a function of time for a) $k$-SWN (solid) and SWN (dashed) , and b) SF networks

- Uniform: As in the previous case, with a uniform distribution ranging from 0 and the number of messages in the node.

They tend to give qualitatively similar results to the quadratic. Discuss cubic case

-Comparison with errors in the propagation of information

[1] S. Boccaletti , V. Latora, Y. Moreno, M. Chavez, D.-U. Hwang, Phys. Rep. 424, 175308 (2006)
[2] A. Czaplicka, J. A. Holyst , P. M. A. Sloot, Scientific Reports 31223 (2013)

[3] M. Kuperman, G. Abramson, Phys. Rev. Lett. 86, 2909. (2001)

[4] G. Abramson and M. Kuperman Phys. Rev. E 63, 030901R (2001)

[5] M.N. Kuperman Phys. Rev. E 73, 046139 (2006)

[6] M.O. Jackson, B. Rogers, Advan. Theoret. Econ. 7, 113 (2007)

[7] D. López-Pintado, Games and Economic Behavior 62, $573590(2008)$

[8] J. Gómez-Gardees, V. Latora Phys. Rev. E 78, 065102(R) (2008)

[9] L. Huang, K. Park, Y. C. Lai, Phys Rev. E 73, 035103-R (2006).

[10] J.D. Noh, H. Rieger, Phys. Rev. Lett. 92, 118701 (2004). 


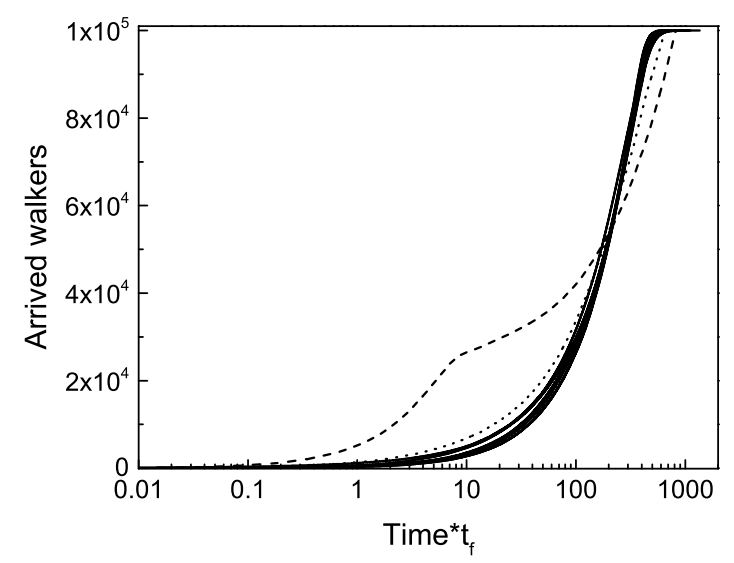

FIG. 8: Collapsed cumulative fraction of arrived agents as a function of time for $k$-SWN, SWN and SF networks. the dashed curve corresponds to a SF network with $a=0.4$

[11] L. Adamic, R.M. Lukose, A.R. Puniyani, B.A. Huberman, Phys. Rev. E 64, 46135 (2001).

[12] B.J. Kim, C.N. Yoon, S.K. Han, H. Jeong, Phys. Rev. E 65, 27103 (2002).

[13] N. T. J. Bailey, The Mathematical Theory of Infectious Diseases and its Applications. (Hafner Press, 1975)

[14] E. M. Rogers, Diffusion of Innovations. (Free Press, New York, 1995)

[15] D. Liben-Nowell, J. Kleinberg, PNAS 105, 4633-4638 (2008)

[16] S.H. Yook,F. Radicchi, H. Meyer-Ortmanns, Phys. Rev.
E 72, 045105-R (2005)

[17] D.J. Watts, S.H. Strogatz, Nature 393, 440 (1998)

[18] M. N. Kuperman, S. Risau-Gusman, Eur. Phys. J. B 62, 233238 (2008)

[19] A. L. Barabási, R. Albert, Science 286, 509 (1999).

[20] R. Xulvi-Brunet, I. M. Sokolov, Phys. Rev. E 70, 066102 (2004)

[21] A. N. Samukhin, S. N. Dorogovtsev, J. F. F. Mendes, Phys. Rev. E 77, 036115 (2008)

[22] A. Czaplicka, J. A. Holyst, P. M. A. Sloot, Sci. Rep. 3, $1223(2013)$

[23] S. Kullback, R. A. Leibler, Annals of Mathematical Statistics 22, 7986 (1951)

[24] A. Barrat, M Weigt, Eur. Phys. J. B. 13, 547560 (2000)

[25] M.E.J. Newman. Phys. Rev. Lett. 89, 208701 (2002)

[26] Wang, H., \& Shang, L. (2015). Physica A: Statistical Mechanics and its Applications, 421, 180-186.

[27] DeGroot, M. H. (1974). Journal of the American Statistical Association, 69(345), 118-121.

[28] Berger, R. L. (1981). Journal of the American Statistical Association, 76(374), 415-418.

[29] Gilardoni, G. L., \& Clayton, M. K. (1993). The Annals of Statistics, 391-401.

[30] Holley, R. A., \& Liggett, T. M. (1975). The annals of probability, 643-663.

[31] Sznajd-Weron, K., \& Sznajd, J. (2000). International Journal of Modern Physics C, 11(06), 1157-1165.

[32] Fazeli, A., \& Jadbabaie, A. (2011, December). In Decision and Control and European Control Conference (CDC-ECC), 2011 50th IEEE Conference on (pp. 23412346). IEEE.

[33] DeMarzo, P. M., Vayanos, D., \& Zwiebel, J. (2003). The Quarterly Journal of Economics, 118(3), 909-968. 


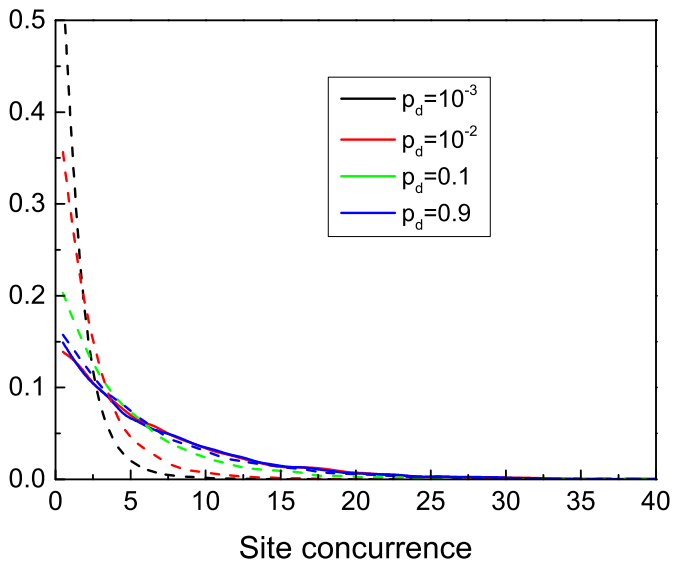

(a)

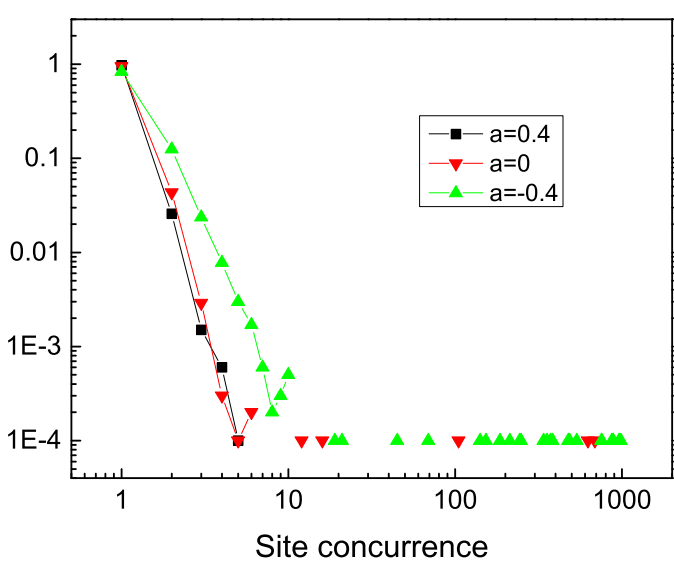

(b)

FIG. 9: Distribution of the number of agents per node when $40 \%$ of packages has been delivered for a) $k$-SWN (solid) and SWN (dashed), and b) SF networks

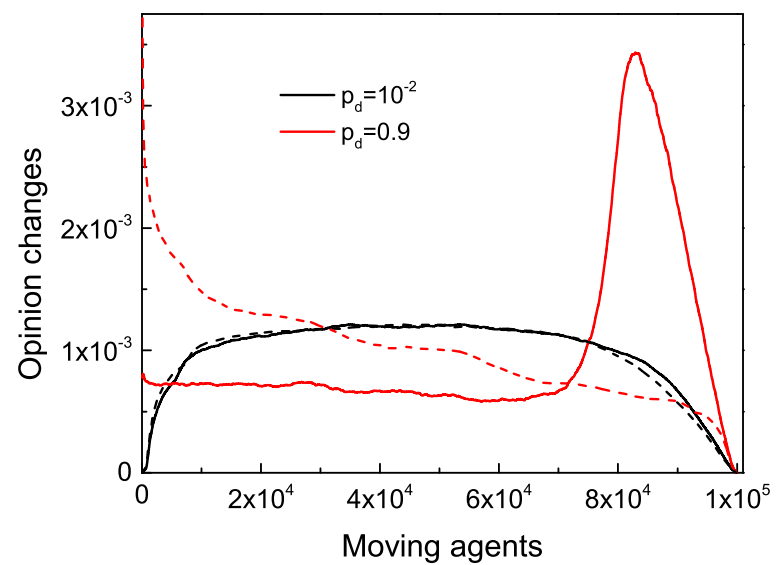

(a)

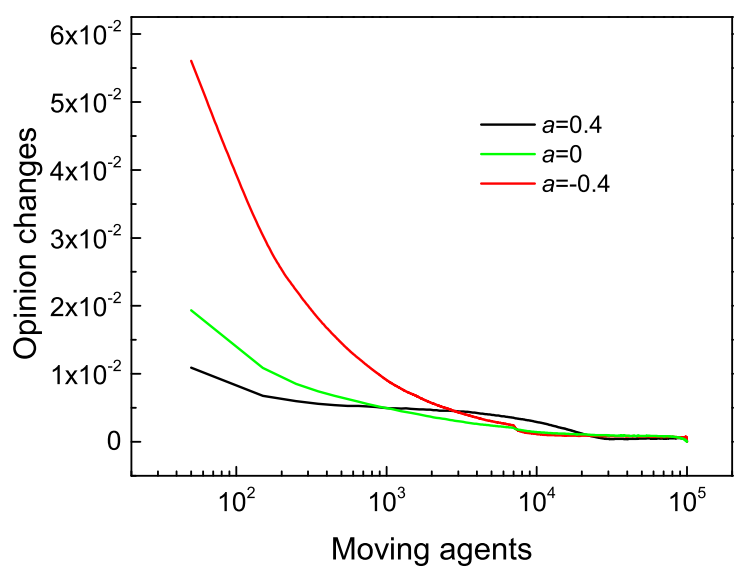

(b)

FIG. 10: Error occurrence as a function of mobile agents for a) $k$-SWN (solid) and SWN (dashed), and b) SF networks 